Research paper

\title{
Quantum dot agglomerates in biological media and their characterization by asymmetrical flow field-flow fractionation
}

\author{
Alexandre Moquin $^{\mathrm{a}, \mathrm{b}, 1}$, Kevin D. Neibert ${ }^{\mathrm{b}, 1}$, Dusica Maysinger ${ }^{\mathrm{b}}$, Françoise M. Winnik ${ }^{\mathrm{a}, \mathrm{c}, *}$ \\ a Department of Chemistry and Faculty Pharmacy, Université de Montréal, Montréal, Canada \\ ${ }^{\mathrm{b}}$ Department of Pharmacology and Therapeutics, McGill University, Montreal, Canada \\ ${ }^{\mathrm{c}}$ WPI International Center for Materials Nanoarchitectonics (MANA), National Institute for Materials Science, Tsukuba, Japan
}

\section{A R T I C L E I N F O}

\section{Article history:}

Received 15 August 2014

Accepted in revised form 13 December 2014

Available online 23 December 2014

\section{Keywords:}

Agglomeration state

Cell uptake

Cell viability

DLS

Asymmetrical flow field-flow fractionation Nanoparticle

Zeta potential

\begin{abstract}
A B S T R A C T
The molecular composition of the biological environment of nanoparticles influences their physical properties and changes their pristine physicochemical identity. In order to understand, or predict, the interactions of cells with specific nanoparticles, it is critical to know their size, shape, and agglomeration state not only in their nascent state but also in biological media. Here, we use asymmetrical flow fieldflow fractionation (AF4) with on-line multiangle light scattering (MALS), dynamic light scattering (DLS) and UV-Visible absorption detections to determine the relative concentration of isolated nanoparticles and agglomerates in the case of three types of semi-conductor quantum dots (QDs) dispersed in Dulbecco's Modified Eagle Media (DMEM) containing 10\% of fetal bovine serum (DMEM-FBS). AF4 analysis also yielded the size and size distribution of the agglomerates as a function of the time of QDs incubation in DMEM-FBS. The preferred modes of internalization of the QDs are assessed for three cell-types, N9 microglia, human hepatocellular carcinoma cells (HepG2) and human embryonic kidney cells (Hek293), by confocal fluorescence imaging of live cells, quantitative determination of the intracellular QD concentration, and flow cytometry. There is an excellent correlation between the agglomeration status of the three types of QDs in DMEM-FBS determined by AF4 analysis and their preferred mode of uptake by the three cell lines, which suggests that AF4 yields an accurate description of the nanoparticles as they encounter cells and advocates its use as a means to characterize particles under evaluation.
\end{abstract}

(c) 2014 Elsevier B.V. All rights reserved.

\section{Introduction}

Luminescent semi-conductor nanocrystals or quantum dots (QDs) are frequently used in the biomedical field for in vitro studies, diagnostics, and small animal imaging, in view of their high quantum yield, size-tunable optical properties, and outstanding photostability $[8,28,13,18]$. They are also used in technological

Abbreviations: AF4, asymmetrical flow field-flow fractionation; ANOVA, analysis of variance; DHLA, dihydrolipoic acid; DLS, dynamic light scattering; DMEM, Dulbecco's Modified Eagle Media; DWF, differential weight fraction; FBS, fetal bovine serum; GFFAA, graphite furnace flame atomic absorption; ICP-MS, inductively coupled plasma-mass spectrometry; MALS, multiangle light scattering; MPA, mercaptopropionic acid; PEG, poly(ethylene glycol); QDs, quantum dots; ROS, reactive oxygen species; SEC, size exclusion chromatography; TEM, transmission electron microscopy.

* Corresponding author. Department of Chemistry and Faculty Pharmacy, Université de Montréal, CP 6128 Succursale Centre Ville, Montréal, QC H3C 3J7, Canada. Tel.: +1 (514) 3405179.

E-mail address: francoise.winnik@umontreal.ca (F.M. Winnik).

1 These authors contributed equally. devices, most notably in solar cells and light-emitting devices. The expansion of QDs from the biologists' laboratory to consumer products raises issues on their toxicity. Previous studies indicate that causal factors to their cytotoxicity include the release of toxic ions, primarily cadmium ions $\left(\mathrm{Cd}^{2+}\right)$, the formation of reactive oxygen species (ROS), and DNA damage [18]. In order to understand the various facets of the damaging potential of QDs upon inadvertent exposure, it is necessary to understand their pathways in and out of cells. To gather meaningful cell trafficking data, one needs an accurate description of QDs as they encounter cells, most importantly their size and surface chemistry. The dynamic interplay between synthetic nanoparticles, such as QDs, and biological components is often overlooked, which has created significant confusion in the literature. It is now recognized that nanoparticle agglomeration is a significant issue in conducting toxicity measurements and interpreting the results.

The determination of the size of nanoparticles in cells and in biological fluids is a challenging task. Confocal fluorescence microscopy is the tool of choice to follow the fate of luminescent 
nanoparticles in live cells. Unfortunately, the spatial resolution of this technique is not sufficient to determine the particle size accurately $[9,21]$. Transmission electron microscopy (TEM) has a spatial resolution well suited to measure the size of metallic or semiconductor in cells [29]. This technique has been used to monitor the location and agglomeration status of nanoparticles in cells that, indeed, had to be fixed prior to TEM imaging and could not be used in further biological studies $[30,50,46]$. Given these limitations, it is desirable at least to know the size of nanoparticles in relevant biological milieus, such as cell media and serum. From the viewpoint of analytical chemistry, this measurement proves to be difficult. Dynamic light scattering (DLS) is the standard method to determine the size (hydrodynamic diameter, $D_{h}$ ) of nanoparticles, within $\sim 5 \mathrm{~nm}$ to $<1.0 \mu \mathrm{m}$ in diameter, dispersed in water or saline [17]. Recent work on the analysis of nanoparticles, such as $\mathrm{CeO}_{2}$, $\mathrm{Fe}_{2} \mathrm{O}_{3}, \mathrm{TiO}_{2}$, and QDs $[7,20]$, gives convincing evidence that DLS suffers from major shortcomings in the analysis of nanoparticles in complex media. Techniques other than light scattering, such as analytical ultracentrifugation [38] and disk centrifugation [11,39] have been applied successfully to study the size of nanoparticles in media in the presence or absence of proteins, but their use is still limited to a few laboratories.

It would be useful to use a standard analytical tool, such as size exclusion chromatography (SEC) with multiangle laser light scattering (MALS) detection, to measure the size and size distributions of mixtures of primary nanoparticles, aggregates, and agglomerates that form in biological system. Standard SEC is not suitable here since the mixture components tend to adsorb irreversibly on most solid supports employed as column packing materials. Asymmetrical flow field-flow fractionation (AF4) $[40,12,14]$ yields size data similar to those recovered by SEC, but since the separation takes place in a solvent-filled channel, irreversible analyte adsorption during separation is easily prevented. AF4 is a mild separation technique that permits the quantitative determination of the size and concentration of particles ranging in size from a few nanometers to several microns. It has been used to analyze QDs $[1,49,35]$, gold $[4,5,37,49,47]$, and silver nanoparticles/clusters in buffers $[33,16]$.

The objectives of the work described here were (i) to validate the use of AF4 as a technique to determine the size and agglomeration status of QDs in biological milieus and (ii) to demonstrate that the data retrieved by AF4 accurately describe the size of nanoparticles as they encounter cells prior to internalization and accounts for their in vitro cytotoxicity. To achieve this goal, we devised a three-pronged approach. First, we analyzed by AF4 the size of QDs in cell media with and without serum, paying attention to the effects of ligand chemistry and incubation time on the agglomeration status of QDs. Second, we examined the cellular internalization of the same QDs in the presence of pharmacological inhibitors known to block specific nanoparticle entry modes. Third, we assessed the cytotoxicity of the QDs in three cell types, N9 microglia, human hepatocellular carcinoma cells (HepG2) and human embryonic kidney cells (Hek293). The combined cellular studies suggest that AF4 is a robust technique to gather biologically-relevant data on the agglomeration of QDs and, presumably other nanoparticles, in complex media.

\section{Materials and methods}

\subsection{Materials}

Water was deionized using a Millipore Milli- ${ }^{\circledR}{ }^{\circledR}$ system (Billerica, MA, USA). All chemicals were purchased from Sigma-Aldrich (St. Louis, MO, USA), unless specified otherwise. Dihydrolipoic acid (DHLA) was prepared by reduction of thioctic acid following a known procedure [6]. $\alpha$-Carboxyl- $\omega$-mercapto poly(ethylene gly$\mathrm{col}$ ) (HS-PEG-COOH, $M_{\mathrm{w}} 5000 \mathrm{Da}$ ) was obtained from Iris Biotech (Marktredwitz, Germany). Chemicals were used without further purification unless otherwise stated. TOP/TOPO-coated $\mathrm{CdSe}(\mathrm{CdZnS})$ core-shell QDs were synthesized and purified by a protocol described in detail elsewhere [34]. They were stored as a suspension in chloroform prior to use. Mercaptopropionic acid (MPA) and dihydrolipoic acid (DHLA)-modified QDs were prepared as described previously [1]. QD-PEG-COOH nanoparticles were prepared from TOP/TOPO-coated CdSe(CdZnS) core-shell QDs [26]. Dulbecco's Modified Eagle Media (DMEM) containing 10\% of fetal bovine serum (DMEM-FBS) and 1\% penicillin-streptomycin was obtained from Gibco (Life Technologies, Carlsbad, CA, USA). Optima $^{\mathrm{TM}}$ grade nitric acid was obtained from Thermo Fisher Scientific (Waltham, MA, USA).

\subsection{Instrumentation}

UV-Vis absorbance spectra were recorded with an Agilent diode array spectrometer model 8452 A (Agilent Technologies, Santa Clara, CA, USA). Luminescence spectra of QDs were recorded on a Varian Cary Eclipse fluorescence spectrometer (Agilent Technologies, Santa Clara, CA, USA) ( $\lambda_{\text {ex }} 365 \mathrm{~nm}$; excitation and emission slits: $5 \mathrm{~nm}$ ). TEM studies were performed on a FEI Tecnai 12 (Hillsboro, OR, USA), $120 \mathrm{kV}$ transmission electron microscope equipped with an AMT XR80C CCD Camera System. Samples were deposited from dispersions in water or chloroform onto a Formvar-coated copper grid or a polymer-coated carbon grid, respectively. The $\zeta-$ potential and hydrodynamic diameter $\left(D_{h}\right)$ of QD dispersions in deionized water or PBS buffer ( $\mathrm{pH}$ 7.4) were measured on a Malvern Zetasizer Nano ZS (Worcestershire, UK). This instrument operates at a wavelength of $633 \mathrm{~nm}$ at a laser power of $4 \mathrm{~mW}$ and scattering angle of $173^{\circ}$. The zeta-potential measurements were run at $25^{\circ} \mathrm{C}$ after $120 \mathrm{~s}$ of equilibration. The number of subruns was determined automatically by the instrument with a minimum of 10 and a maximum of 100 subruns. The measurements were repeated 3 times per sample. The $Z$-average size value was calculated by the methods of cumulants, as implemented in the Malvern software.

An asymmetrical flow field-flow fractionation (AF4) system (AF 2000 MT, Postnova Analytics, Salt Lake City, USA) with a channel thickness of $350 \mu \mathrm{m}$ and controlled by the AF2000 ${ }^{\circledR}$ Control software (Postnova Analytics, Salt Lake City, USA) was used. The cross-flow was generated by Khloen syringe pumps (Postnova Analytics) while the axial and focusing flows were delivered by isocratic pumps (PN1130, Postnova Analytics). The system was connected to a UV-Vis variable wavelength spectrophotometric detector (SPD-20A, Postnova Analytics), a fluorescence detector (RF-10AXL, Postnova Analytics), a multi-angle light scattering (MALS, Dawn Heleos 8+, Wyatt Technology, Santa Barbara, USA), and a dynamic light scattering (DLS) detector (Wyatt QELS, Wyatt Technology) which is an add-on unit connected to the $108^{\circ}$ angle of the MALS Dawn Heleos 8+ detector. The MALS was equipped with a $\mathrm{K} 5$ cell and a GaAs laser operating at $658 \mathrm{~nm}$. It takes measurements at $1 \mathrm{~s}$ intervals for the MALS and the DLS. The UV, MALS and DLS signals were simultaneously recorded as plots of detector signal versus time (fractograms). Data collection and analysis were done using the ASTRA $^{\circledR}$ software version 6.1.1.17 provided by Wyatt Technology. A regenerated cellulose membrane (Z-MEMAQU-627, $M_{\mathrm{w}}$ cut-off $10 \mathrm{kDa}$, Postnova Analytics) was used for the three types of QDs.

\subsection{AF4 Fractionation method}

The carrier medium ( $1 \mathrm{mM}$ phosphate buffer, $\mathrm{pH}$ 7.4) was filtered through a $0.1 \mu \mathrm{m}$ Whatman ${ }^{\circledR}$ filter prior to use (GE 
Healthcare Life Sciences, Pittsburgh, PA, USA). After flow equilibration, the sample was injected with a flow rate of $0.2 \mathrm{~mL} / \mathrm{min}$ (injection loop volume: $21.5 \mu \mathrm{L}$ ), followed by a 5 -min focusing period with a cross-flow rate of $1.2 \mathrm{~mL} / \mathrm{min}$ and a detector flow rate of $0.3 \mathrm{~mL} / \mathrm{min}$. Following a $1 \mathrm{~min}$ transition, the cross-flow rate was decreased linearly from $1.2 \mathrm{~mL} / \mathrm{min}$ to $0 \mathrm{~mL} / \mathrm{min}$ within $20 \mathrm{~min}$. The elution was continued for 10 -min without cross-flow to allow elution of larger particles (size $>60 \mathrm{~nm}$ ). The detector flow rate was kept at $0.3 \mathrm{~mL} / \mathrm{min}$ throughout. The detection of the eluted fractionated QDs/larger agglomerates was performed sequentially by UV absorbance at $280 \mathrm{~nm}$, fluorescence $\left(\lambda_{\mathrm{ex}} 365 \mathrm{~nm}, \lambda_{\mathrm{em}}\right.$ $635 \mathrm{~nm}$ ), MALS, and DLS. The $Z$-average effective spherical hydrodynamic radius of the eluting particles/agglomerates was determined by DLS based on cumulant analysis of the scattered intensity correlation functions measured across each eluting band. Each fractogram presented is representative of a triplicate sample. A calibration of the instrument with QD-DHLA samples of increasing concentration was performed first in order to verify that there was no sample loss due to irreversible interactions with the ultrafiltration membrane used as the channel wall, and that the signal at the detector was proportional to the concentration of the sample (Fig. S6).

Particles injected in the AF4 channel are subjected simultaneously to the longitudinal carrier flow and the applied field that acts perpendicularly to the length of the channel and causes particles to move toward the accumulation wall. Under these conditions, smaller particles travel faster than larger ones, resulting in size fractionation of the sample injected. The eluting fractions are analyzed first by a UV/Vis detector acting as the concentration detector and, second, by MALS and DLS detectors that provide, respectively, the mass and the hydrodynamic diameter $\left(D_{h}\right)$ of the particles as they elute, yielding traces, or fractograms, of the $\mathrm{UV} / \mathrm{Vis}$ absorbance (proportional to the concentration) vs time and $D_{h}$ vs time, respectively.

The overall size distribution of the eluting particles can be presented as the differential weight fraction (DWF), which provides a visual representation of the weight fraction of a sample within a certain size range. The total mass of the eluting sample is calculated as the sum of the mass of each fraction using concentrations obtained from the UV-Vis absorbance elution data, while the hydrodynamic size of each fraction is measured by the DLS detector. The hydrodynamic sizes and corresponding concentrations of the fractions are sorted by ascending size to determine the cumulative distribution (sum of all the concentrations up to the specified particle size). The DWF of the sample is calculated by taking the differential of the cumulative distribution. The data are normalized by multiplying the sum of the concentrations by the volume of the eluting fraction, then dividing by the total mass of the sample. The DWF size distributions were plotted using the Astra ${ }^{\circledR}$ software from the concentration data given by the UV-Vis detector and the size data given by the DLS detector.

\section{4. $Q D$ dispersions in cell culture medium with/without serum}

For AF4 analysis, suspensions of QDs (absorbance $\sim 0.1$ at 590 $600 \mathrm{~nm}$ ) in DMEM and DMEM containing 10\% FBS (DMEM-FBS) were prepared by dilution of $\mathrm{QD}$ stock solutions in deionized water. The QD concentration in the final dispersions was calculated using an empirical correlation relating the optical absorbance (wavelength, intensity) with the particle size and concentration, respectively [48]. The solutions were diluted to a concentration of $200 \mathrm{nM}$ of QDs in either water, DMEM, or DMEM-FBS. For time-dependent AF4 measurements, the QDs were treated with DMEM or DMEM-FBS and incubated at $37{ }^{\circ} \mathrm{C}$. Aliquots taken from the incubating dispersions at various times ( $5 \mathrm{~min}$ to $24 \mathrm{~h}$ ) were injected in the AF4 instrument without further treatment.

\subsection{Cell culture}

Murine microglia cells (N9) were provided by P. Séguéla (Montreal Neurological Institute, Montreal, QC, Canada). Human embryonic kidney cells (Hek293) and human hepatocellular liver carcinoma cells (HepG2) were purchased from ATCC (Manassas, VA, USA) (CRL-1573 and HB-8065, respectively). All cell lines were maintained at $37^{\circ} \mathrm{C}$ under a $5 \% \mathrm{CO}_{2}$ humidified atmosphere in phenol-free Dulbecco's Modified Eagle Media (DMEM, Gibco) containing $10 \%$ fetal bovine serum (FBS, Gibco) and $1 \%$ penicillinstreptomycin (P/S, Gibco). Prior to all cell treatments, cell culture media was aspirated and the cells were washed with $500 \mu \mathrm{L}$ PBS. Details for the drug and QD treatments, confocal microscopy, spectrofluorimetry and cadmium determinations are provided in the supporting information.

\subsection{Cell viability assay}

N9 cells, Hek293 and HepG2 cells were seeded at 100,000 cells/ well in 24-well plates (Sarstedt, Nümbrecht, Germany). Cells were treated with cell culture media (DMEM or DMEM-FBS) containing QDs (100 nM) or pre-agglomerated QDs (100 nM) for 1-24 h. Following QD treatment, the cells were washed and incubated with Hoechst $33258\left(10 \mu \mathrm{M}\right.$, Molecular Probes ${ }^{\circledR}$, Life Technologies, Carlsbad, CA, USA) for 10 min for nuclear staining. The stained cells were then washed with $500 \mu \mathrm{L}$ PBS and fixed with $4 \%$ paraformaldehyde (Thermo Fisher Scientific). Fluorescence images were acquired with a Leica DFC350FX monochrome digital camera (Leica Microsystems, Wetzlar, Germany) connected to a Leica DMI4000B inverted fluorescence microscope with $\lambda_{\text {ex/em }}=352 / 462 \mathrm{~nm}$ filters using $10 x$ magnification. Cells were counted using Image ${ }^{\circledR}$ from representative images acquired from at least 3 independent experiments of triplicates.

Experimental procedures for the determination of QD uptake by flow cytometry (FACS), the treatment of cells with pharmacological inhibitors, the determination of QD uptake by spectrofluorimetry, the detection of $\mathrm{QD}$ internalization by confocal microscopy, the quantitative analysis of $\mathrm{Cd}^{2+}$ in QD-treated cells by graphite furnace flame atomic absorption (GFFAA) and the quantitative analysis of $\mathrm{Cd}^{2+}$ in cell culture media by inductively coupled plasmamass spectrometry (ICP-MS) are given as supporting information.

\subsection{Statistical analysis}

Data were analyzed using SYSTAT ${ }^{\circledR} 10$ (Cranes Software International, San Jose, CA, USA). Statistical significance was determined by analysis of variance (ANOVA) followed by post hoc, Dunnett's test, independent $t$-test or by one sample $t$-test were specified. Significant differences are indicated by ${ }^{*} p<0.05,{ }^{* *} p<0.01$, and ${ }^{* * *} p<0.001$.

\section{Results}

\subsection{Characterization of $Q D S$}

The QDs were prepared by conventional ligand exchange, starting with $\mathrm{CdSe}(\mathrm{CdZnS})$ nanocrystals, obtained according to standard protocols [34], subsequently coated with mercaptopropionic acid (MPA), dihydrolipoic acid (DHLA), or $\alpha$-carboxyl- $\omega$-mercaptopoly(ethylene glycol) (HS-PEG-COOH), all of which are commonly used for in vitro studies [6,26] (Fig. 1A). TEM revealed that the $\mathrm{CdSe}(\mathrm{CdZnS})$ nanoparticles have a diameter around $7 \mathrm{~nm}$. The coated QDs have broad absorption in the UV and a sharp luminescence band centered at $\lambda_{\mathrm{em}}=640 \mathrm{~nm}$ (Fig. 1C). 
(A) QD-MPA

QD-DHLA

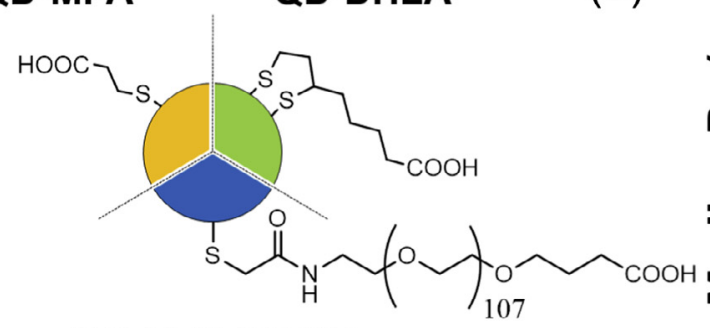

QD-PEG-COOH

(C)

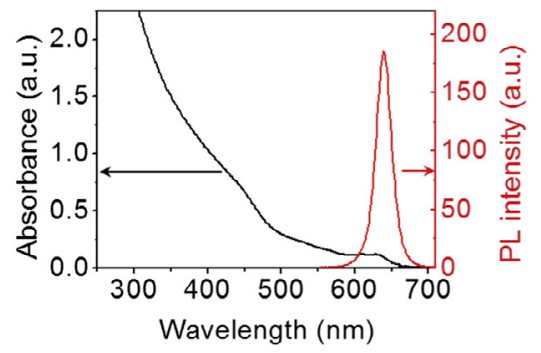

(B)
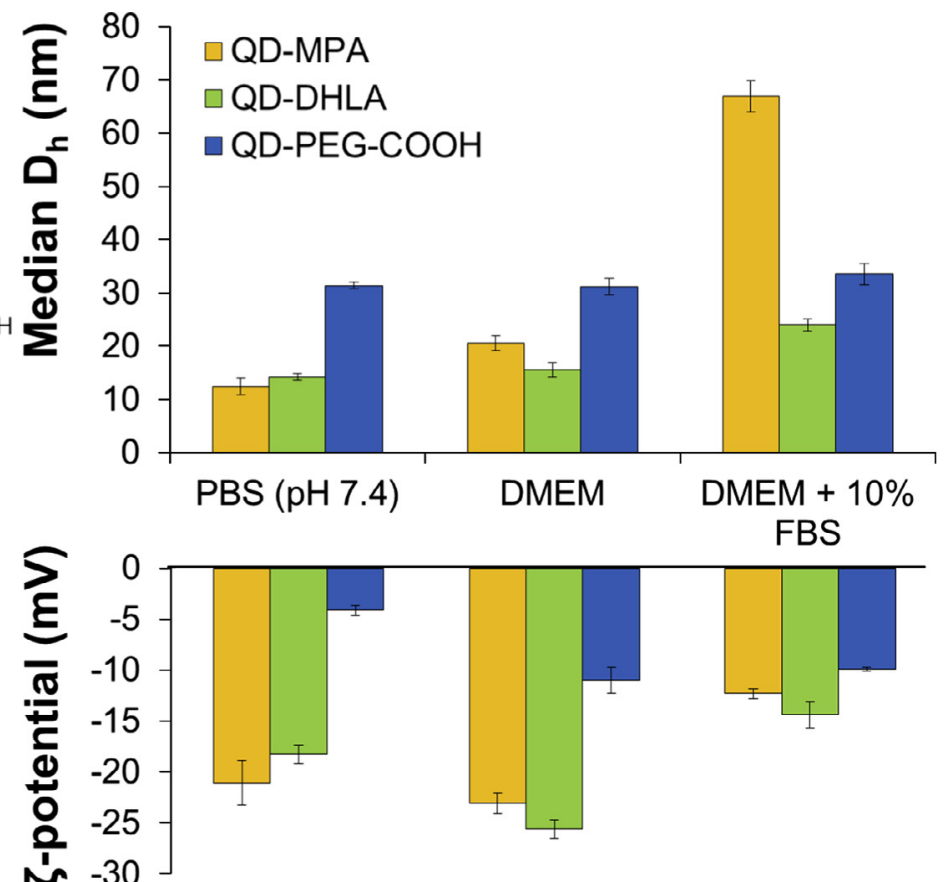

(D)

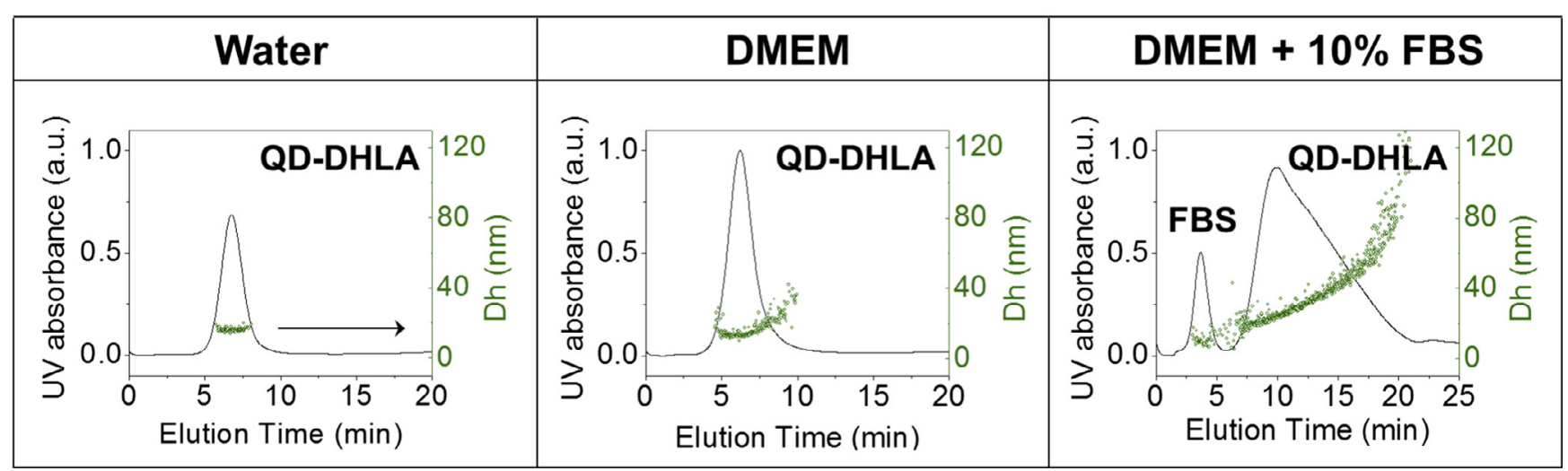

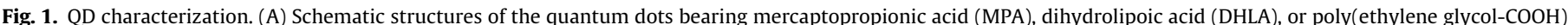

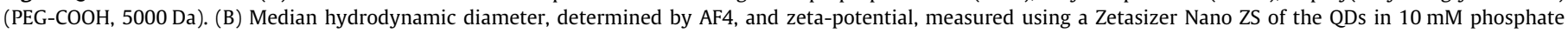

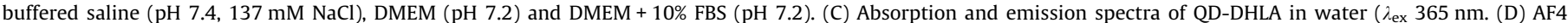

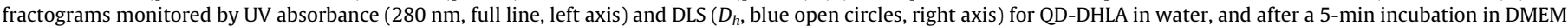
and in DMEM $+10 \%$ FBS. (For interpretation of the references to color in this figure legend, the reader is referred to the web version of this article.)

Dynamic light scattering analysis (batch mode) of the QDs dispersed in PBS ( $\mathrm{pH} 7.4)$ revealed a polydisperse size distribution of nanoparticles, which had hydrodynamic diameters $\left(D_{h}\right)$ of 12.4 , 14.2, and $31.4 \mathrm{~nm}$ for QD-MPA, QD-DHLA, and QD-PEG-COOH), respectively. The $\zeta$-potentials of QD-MPA and QD-DHLA dispersed in PBS were $-21.1 \mathrm{mV}$ and $-18.3 \mathrm{mV}$, respectively, while the PEG-COOH coated QDs were nearly neutral.

The AF4 elution profiles (fractograms) recorded for QD-DHLA dispersed in water and after a 5-min incubation in DMEM or DMEM-FBS are presented in Fig. 1D. In each fractogram, the full line (black, left-hand ordinate) is the elution profile recorded by the UV-Vis detector set at $\lambda_{\mathrm{abs}}=280 \mathrm{~nm}$, while the green dotted line (right-hand ordinate) corresponds to the $D_{h}$ of the nanoparticles eluting within the time spanning the elution peak detected by UV-Vis absorbance. The median $D_{h}$ values retrieved from the fractograms are given in Fig. 1B. The UV-absorbance fractograms feature bands at $\sim 7$ and $\sim 10 \mathrm{~min}$, for QD-DHLA in water and in DMEM (Fig. 1D, left and middle panels). The bands correspond, respectively, to the elution of nanoparticles of $D_{h} \sim 12 \mathrm{~nm}$ and
$24 \mathrm{~nm}$, as determined by DLS (green open circles, Fig. 1D). The fractogram of QD-DHLA incubated with DMEM-FBS (Fig. 1D, right panel) presents two bands: a band at very short time $(3.8 \mathrm{~min}$, $D_{h} 7 \mathrm{~nm}$ ), corresponding to the elution of the proteins present in FBS, and a broad band with a maximum at $\sim 10 \mathrm{~min}$ and a shoulder at long elution times, corresponding to the elution of particles of $D_{h}$ ranging from $\sim 16-20 \mathrm{~nm}$ to nearly $80 \mathrm{~nm}$.

Dispersions of the three batches of QDs in water, DMEM, and DMEM-FBS were analyzed by AF4 after a 24-h incubation. The composition of the samples is presented in Fig. 2 in the form of differential weight fraction plots, which offer a visual representation of the weight fraction of the eluting species within a certain size range. The median diameter of QD-PEG-COOH dispersed in DMEM-FBS undergoes a slight shift to larger values upon incubation, which may be indicative of some level of protein adsorption [42]. In DMEM, QD-MPA form small agglomerates with a median radius of $13.7 \mathrm{~nm}$, whereas in DMEM-FBS large aggregates form, with a median radius of $64.5 \mathrm{~nm}$ and a broad size distribution. QD-DHLA form small agglomerates in DMEM-FBS, similar in size 

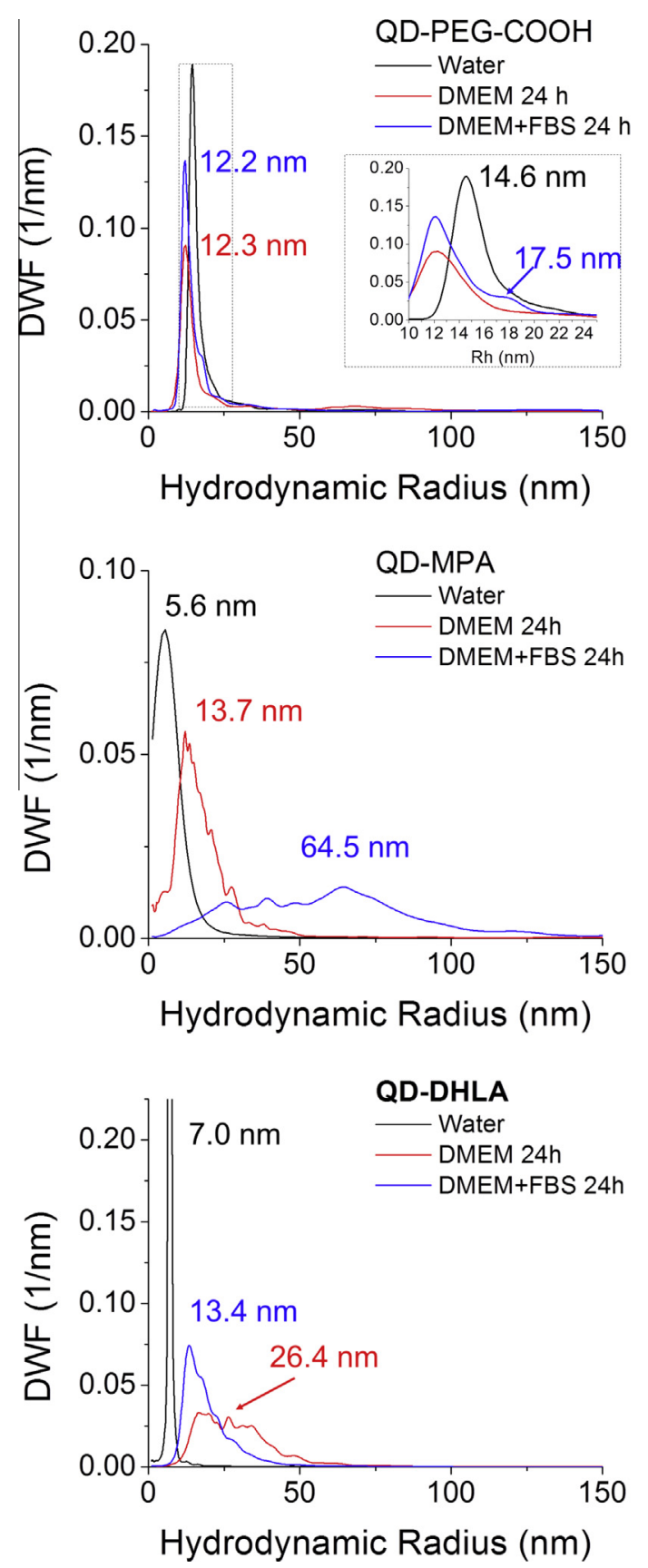

Fig. 2. Size distribution presented as differential weight fraction (DWF) of QD-PEG$\mathrm{COOH}$ (top), QD-MPA (middle), and QD-DHLA (bottom) of the QDs after incubation in deionized water, cell culture media or cell culture media (DMEM) supplemented with $10 \%$ FBS for $24 \mathrm{~h}$. (For interpretation of the references to color in this figure legend, the reader is referred to the web version of this article.)

to the QD-PEG-COOH in the same medium. In DMEM, QD-DHLAs form agglomerates with a median radius of $26.4 \mathrm{~nm}$.

The agglomeration time course over a 24-h incubation for QDs in water, DMEM, and DMEM-FBS is presented in Fig. 3, where we present concentration versus time fractograms of aliquots taken from the dispersions during the incubation. The collected fractograms reveal that the agglomeration of QD-MPA and QD-DHLA in DMEM-FBS is extremely fast ( $<5 \mathrm{~min}$ ) (Fig. $3 \mathrm{~B}$ and C). The size of the QD-DHLA agglomerates formed at $t=5 \mathrm{~min}$ does not change significantly with time (Fig. 3C), whereas in the case of QD-MPA agglomerates grow in size over time (Fig. 3B), particularly over the first hour. QD-DHLA dispersions in DMEM resist agglomeration in the first incubation hour (Fig. 3F); with time, agglomerates form and gradually grow in size. QD-MPA agglomeration occurs gradually over the entire incubation period (Fig. 3E).

\subsection{Toxicity of $Q D s$ to cultured cells}

In their native form, the QDs (100 nM) caused no significant toxicity to cultured N9 microglia, Hek293, and HepG2 cells after $24 \mathrm{~h}$. To investigate the impact of QDs agglomeration status on their cellular uptake and toxicity, the cells were incubated with QD samples subjected to a 24-h pre-incubation in DMEM-FBS prior to use. In the case of N9 cells, QD-MPA and QD-DHLA pre-incubated for $24 \mathrm{~h}$ in DMEM-FBS caused, respectively, $25.4 \pm 4.8 \%$ and $19.3 \pm 5.1 \%$ cell death (Fig. $4 \mathrm{~A}$ ). The enhanced toxicity of pre-incubated QD-MPA, compared to native QD-MPA, may reflect their increased uptake by $\mathrm{N} 9$ cells ( $+36 \%$ after $6 \mathrm{~h}$ ) compared to native QD-MPA (Fig. 4B). Yet, for QD-DHLA, there was no significant difference in the extent of internalization between native and preincubated QD-DHLA (100\% and 108\%, respectively at $6 \mathrm{~h}$ ). Hence, it may be surmised that the agglomeration status of QDs is an important factor as well. QD-PEG-COOH samples caused no significant cytotoxicity after pre-incubation for $24 \mathrm{~h}$ in DMEM-FBS. Increased cytotoxicity of QD-MPA and QD-DHLA after a 24-h preincubation in FBS-DMEM was also observed for liver cells (Fig. 4C), but not for kidney cells (Fig. 4E). As seen in the case of N9 cells, QD-MPA pre-incubated for $24 \mathrm{~h}$ in FBS-DMEM are internalized faster and to a higher extent in the liver cells ( $+33 \%$ after $6 \mathrm{~h}$ ), compared to native QD-MPA (Fig. 4D). The uptake of QDPEG-COOH by liver ( $17 \%$ after $6 \mathrm{~h}$ ) and kidney cells (12\% after $6 \mathrm{~h}$ ) was significantly smaller, compared to QD-MPA (63\% after $6 \mathrm{~h}$ in HepG2 and 95\% after $6 \mathrm{~h}$ in Hek293) or QD-DHLA (95\% after $6 \mathrm{~h}$ in HepG2 and 93\% after $6 \mathrm{~h}$ in Hek293). QD-PEG-COOH exhibited no detectable toxicity to N9, Hek293 and HepG2 cells.

\subsection{Internalization modes of QDs in cultured cells}

To study the preferred endocytotic pathways adopted by QDs bearing different ligands, cells treated for $30 \mathrm{~min}$ with pharmacological inhibitors were exposed to QDs pre-incubated for $24 \mathrm{hrs}$ in DMEM-FBS. The QDs did not interact with the inhibitors used as demonstrated by the AF4 of the QDs in the presence of the inhibitors (Fig. S5). Several modes of QD internalization seem to be at play in microglia and the two human cell lines. The main modes of internalization of agglomerated QDs (QD-MPA and QDDHLA) in microglia are phagocytosis and to a lesser degree macropinocytosis [21], which can be correlated to the formation of agglomerates of these QDs exposed to FBS-DMEM detected by AF4 analysis. Results obtained by cholesterol depletion by MBCD suggest involvement of lipid rafts in all cell types investigated. In Fig. 5, we present confocal fluorescence microscopy observations of N9 microglia cells cultured in DMEM-FBS and DMEM in the presence and absence of the phagocytosis inhibitor SB-203580 and treated for $1 \mathrm{~h}$ with $100 \mathrm{nM}$ QDs. They illustrate the involvement of phagocytosis in the internalization of agglomerated QDs.

Quantitative determination of QD uptake in cells was retrieved from the measurement of the $\mathrm{Cd}^{2+}$ concentration within $\mathrm{N} 9$ microglia after treatment with QDs under various conditions. The results are presented graphically on the right-hand side of Fig. 5 for cells pre-incubated, or not, with SB-203580. In all cases the intracellular $\mathrm{Cd}^{2+}$ concentration was the highest for cells treated with QD-MPA. Moreover, intracellular $\left[\mathrm{Cd}^{2+}\right]$ was significantly reduced in cells treated with QD-MPA that were cultured in DMEM-FBS and pretreated with the phagocytosis inhibitor, confirming the visual assessments of micrographs and the fluorimetric data presented in Fig. S7. We determined also the concentration of free $\mathrm{Cd}^{2+}$ that may have leached from the QDs upon prolonged 
(A)

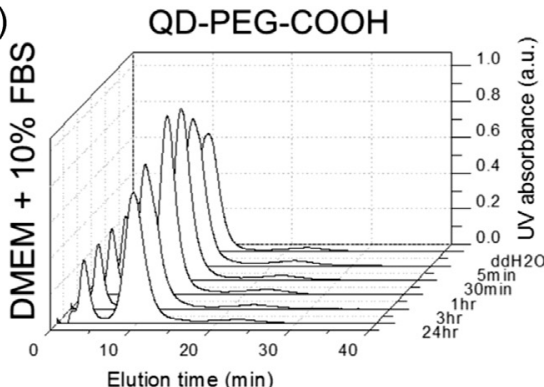

(B)

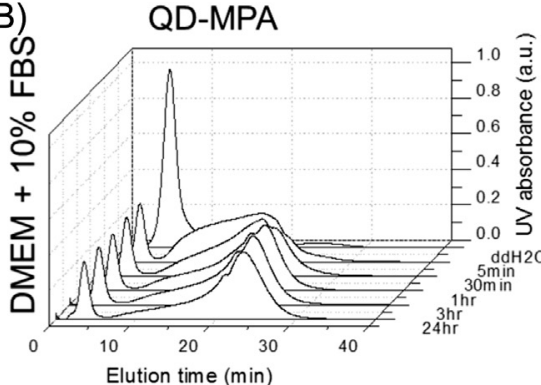

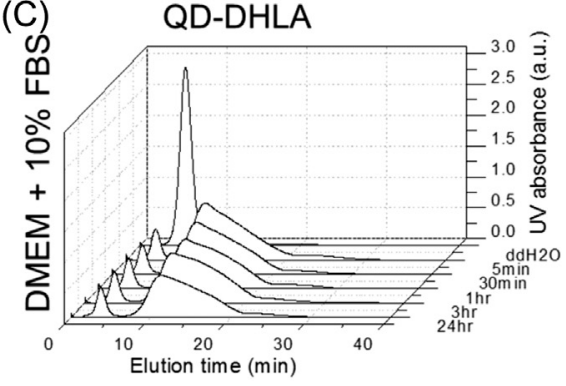

$(\mathrm{F})$

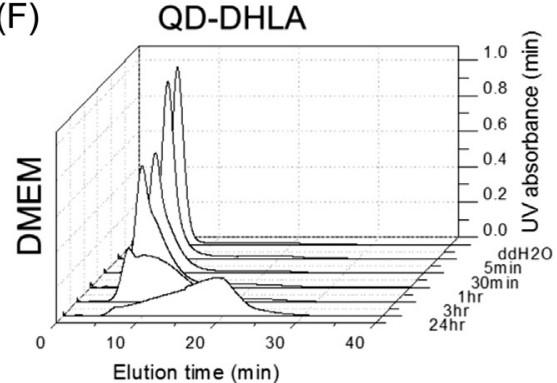

Elution time $(\mathrm{min})$
(D)

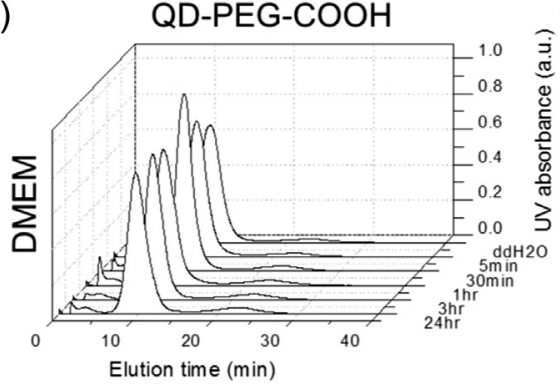

$(\mathrm{E})$

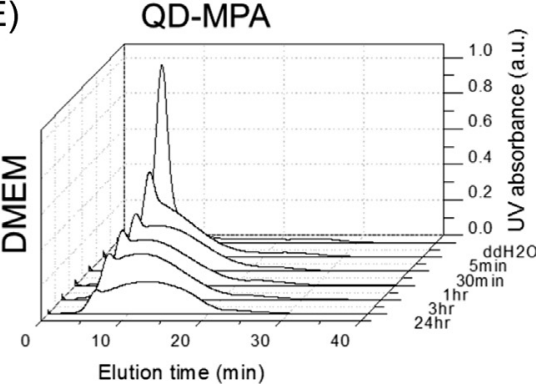

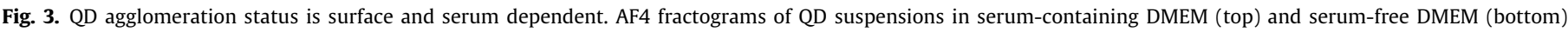

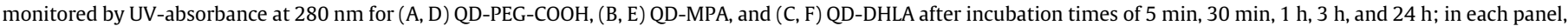
the backmost fractogram corresponds to the elution of the primary QDs in deionized water.

incubation, in order to confirm the validity of the quantitative determination of $\mathrm{QD}$ uptake. The concentrations of $\mathrm{Cd}^{2+}$ in serum-containing DMEM after a 48-h incubation of QD-MPA and QD-PEG-COOH were $19.95 \mathrm{ppm}$ and $104.02 \mathrm{ppm}$, respectively. These values are significantly lower that the $\mathrm{Cd}^{2+}$ values, $14,766.7 \mathrm{ppm}$ and 58,297.9 ppm, obtained by digestion of cells treated with $100 \mathrm{nM}$ of QD-MPA and QD-PEG-COOH, respectively.

\section{Discussion}

\subsection{Nanoparticles in complex cell culture media}

$\mathrm{CdSe} / \mathrm{CdZnS}$ nanocrystals of identical size but coated with three different negatively charged ligands, mercaptopropionic acid (MPA), dihydrolipoic acid (DHLA) and carboxylated-poly(ethylene glycol) (PEG-COOH), displayed distinct behaviors with respect to agglomeration in cell cultured media. The QD-PEG-COOH showed no sign of agglomeration, neither in DMEM, nor in DMEM-FBS. The PEGylated ligand provides excellent dispersion stability to the QDs due to the synergy of the steric effect, attributed to the hydrated PEG-chain [10] and the electrostatic repulsion among negatively-charged end groups. The QDs bearing shorter ligands, MPA or DHLA, tend to agglomerate, but not under the same conditions. QD-MPAs form small agglomerates in DMEM and much larger ones in DMEM-FBS, whereas QD-DHLAs exhibit the opposite behavior: They agglomerate to a much larger extent in DMEM than in DMEM-FBS. MPA ligands are attached to the QD surface through monodentate thiol bonds, while the DHLA linkage to the QD surface is bidentate. The ligand packing density is higher in the case of QD-MPA, compared to QD-DHLA [25,51], which may account for the enhanced stability of QD-MPA in DMEM, compared to QD-DHLA. The low packing density of DHLA leaves space available for the adsorption of proteins, favoring the formation of small QDDHLA agglomerates surrounded by a protein corona that prevents further agglomeration. The role of serum proteins in preventing agglomeration of inorganic nanoparticles was observed previously with diverse materials, such as iron oxide [44], zinc oxide [44], tita- nium dioxide [3], and tungsten carbide [27], in the presence of serum proteins.

\subsection{Implications of $Q D$ agglomeration on cell culture studies}

Most nanoparticles, except small NPs coated with steric stabilizers, such as PEG ligands, will agglomerate in cell culture media within the time scale of cell culture experiments, as shown here and in previous studies [29,2]. In most cases, particularly for NPs bearing short ligands, agglomeration must be taken into consideration in the design and analysis of cellular experiments. Our study demonstrates that the agglomeration status of QDs is a key determinant of the mode of QD entry in the cell. Phagocytosis is more efficient for agglomerates than for single nanoparticles, in agreement with previous studies on the internalization of QDs and metal oxide nanoparticles $[15,44,11,31]$.

Although in this current study we have focused on the agglomeration status of QDs, AF4 could also be applied to other types of nanoparticles, of inorganic or organic composition, to study their size distribution when placed in biological media. Depending on the size, density, or surface property of the initial nanoparticles, the agglomeration kinetics may be significantly different. AF4 online with DLS is limited by the size range it can effectively characterize because of the limitation of the DLS detector $(<1000 \mathrm{~nm})$. However, AF4 can also be calibrated with standard beads of known sizes to study samples larger than the micron. For those sizes, the steric mode of AF4 can be applied.

\section{3. $Q D$ agglomeration and cytotoxicity}

This study indicates that, overall, QD agglomerates, for example those formed by pre-incubation of QD-MPA in DMEM-FBS, were more cytotoxic than single QDs dispersed in water tested immediately after preparation (native QDs) or QD-PEG-COOH. Although this trend has been reported previously, its origin remains controversial [22,19,32]. Agglomerated QDs are internalized primarily via phagocytosis, hence their cellular pathway and fate differ from that of small individual nanoparticles, with possible implication on 


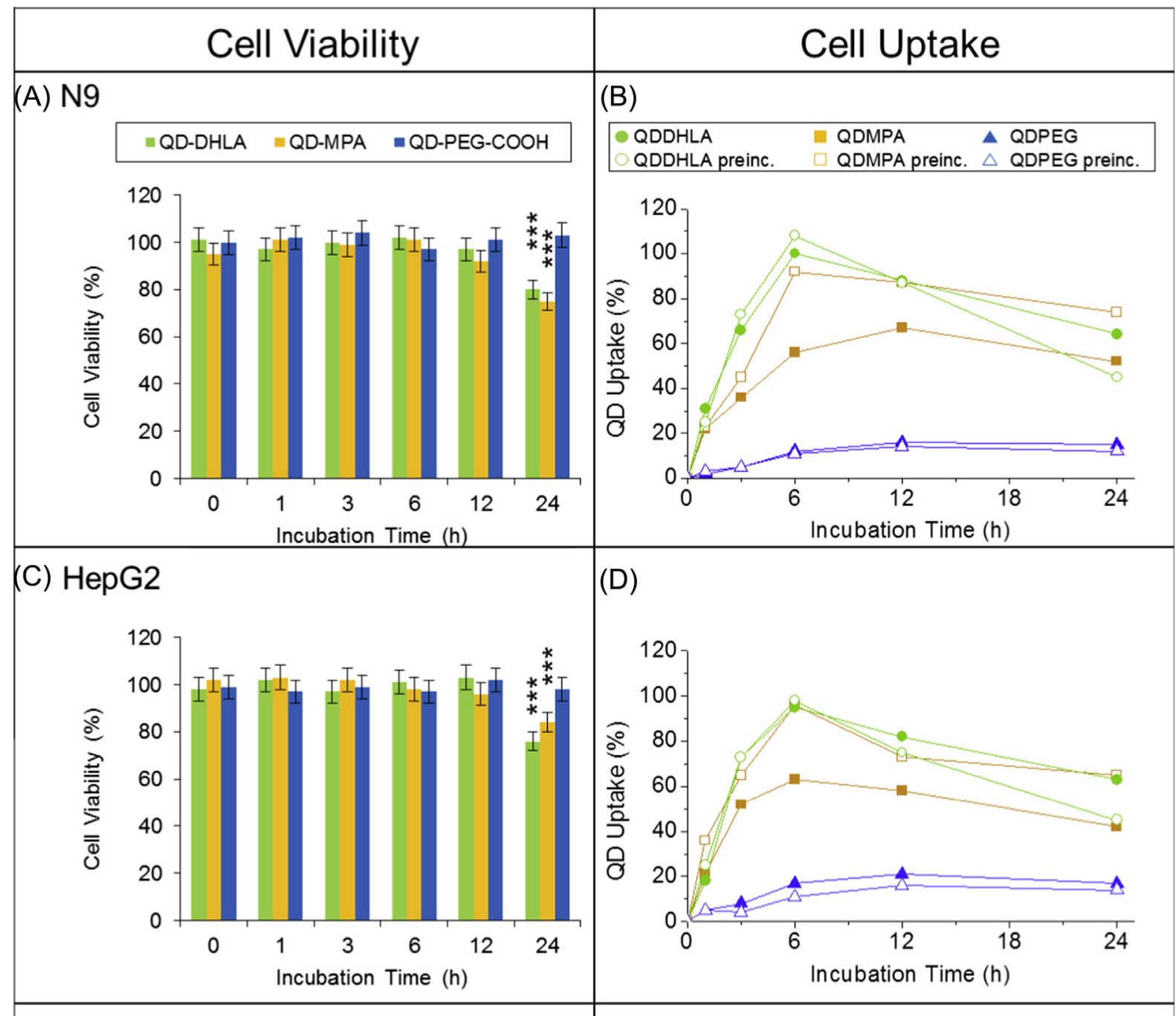

(E) Hek293

( $F)$
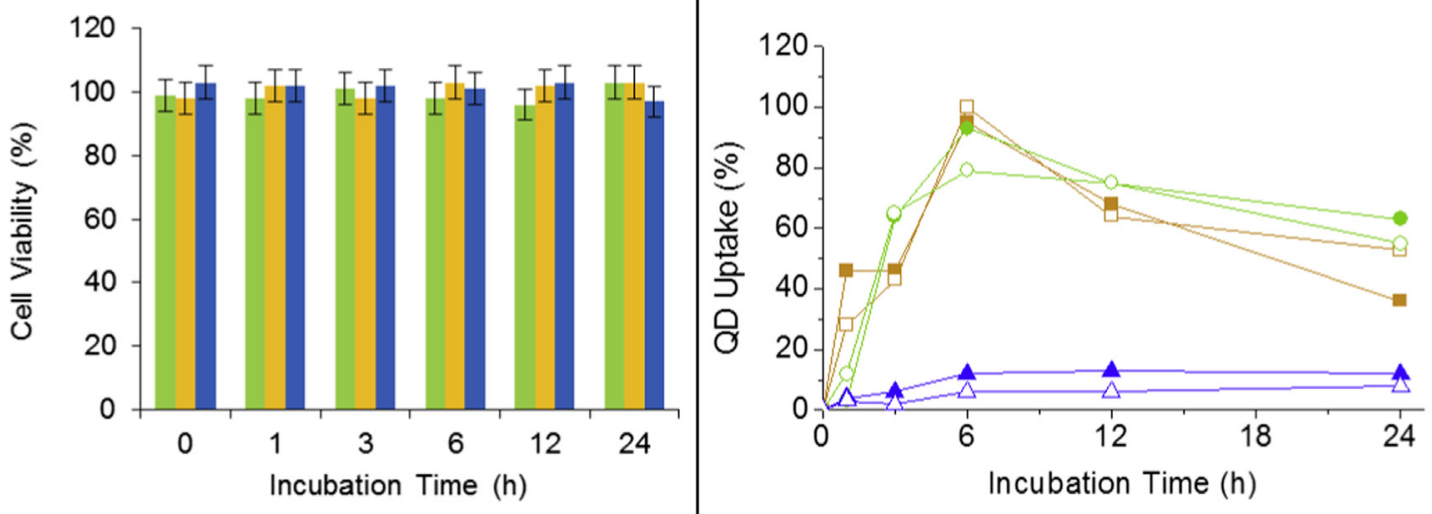

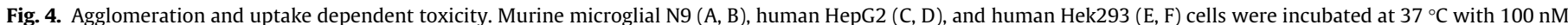

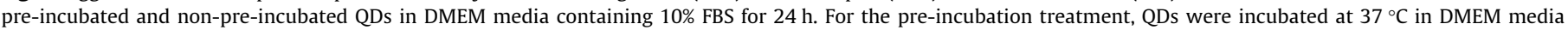

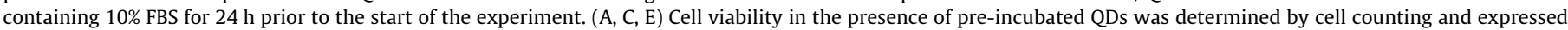

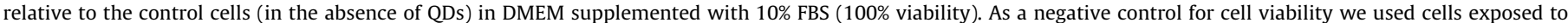

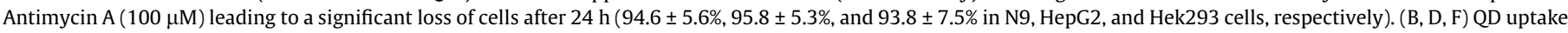

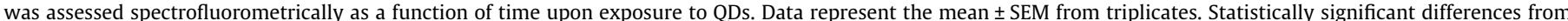

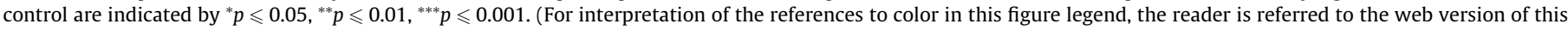
article.)

their toxicity. The agglomeration of QDs in serum is facilitated by adsorption of serum proteins. Although the exact composition of the protein layer surrounding QD agglomerates is not fully defined at present [41-43], it is expected that the proteins unfold upon adsorption, which may have deleterious effects [24]. Adsorption may inactivate enzymatic or signaling functions of the protein, 

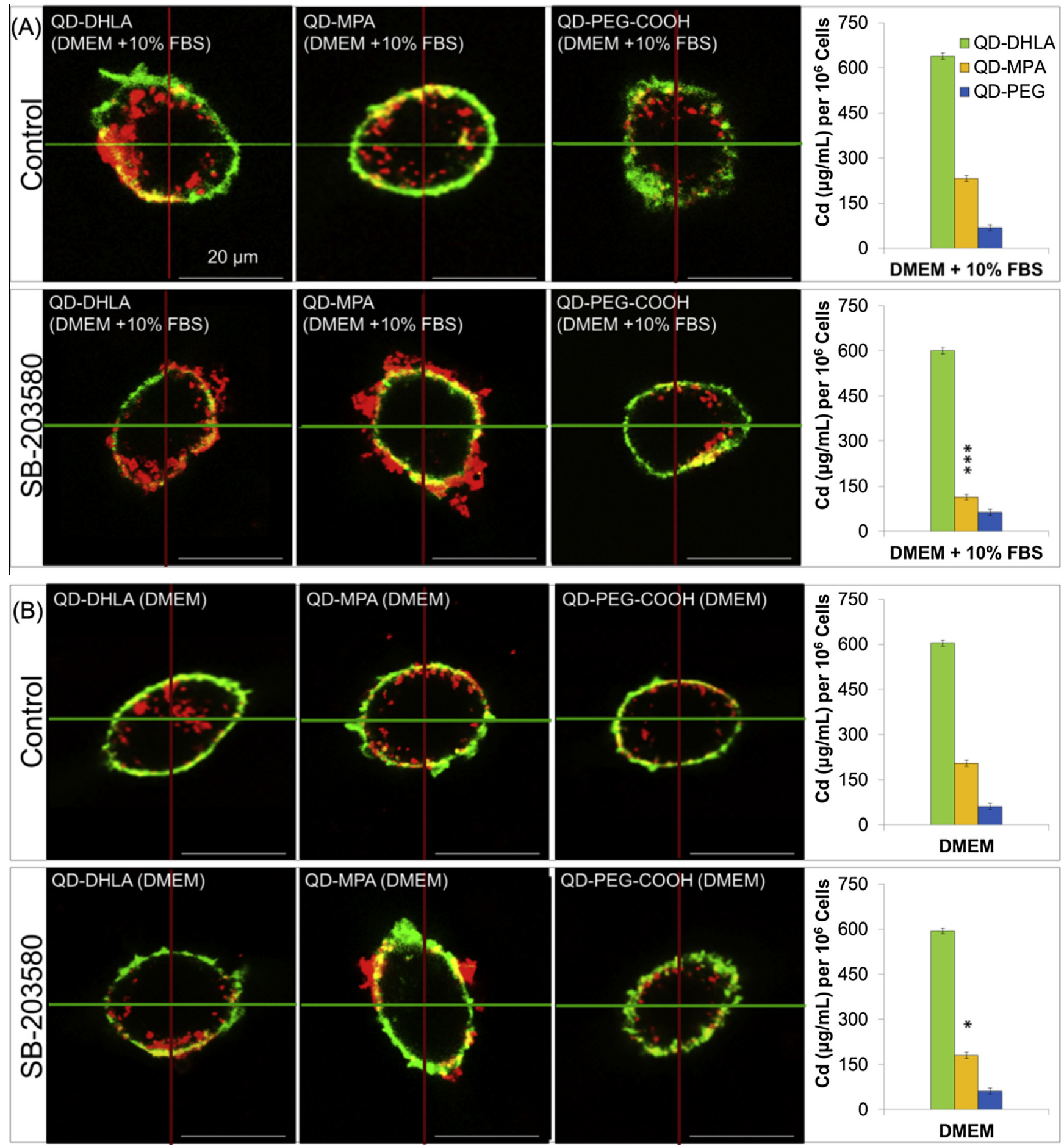

Fig. 5. Live cell confocal micrographs of N9 microglia cells incubated with QD-DHLA, QD-MPA and QD-PEG-COOH (100 nM; $1 \mathrm{~h}$ ) in A) serum-containing media (DMEM +10\% FBS) or (B) serum-free media. The cells were either pre-treated or not with SB-203580 $(10 \mu \mathrm{M})$, inhibitor of phagocytosis. The plasma membrane is labelled in green (PKH) and QDs are visible in red. Representative pictures are selected from triplicates of at least 3 independent experiments. Scale bars represent $20 \mu \mathrm{m}$. The extent of QD uptake was assessed by intracellular cadmium content (GFAAS) after exposure to three differently coated QDs $(100 \mathrm{nM}, 1 \mathrm{~h})$. The data represent the means \pm SEM $(n=9)$. ${ }^{*} p<0.05$, ${ }^{* *} p<0.01,{ }^{* * *} p<0.001$. (For interpretation of the references to color in this figure legend, the reader is referred to the web version of this article.)

provoke immune responses, or trigger irreversible aggregation of soluble proteins $[23,45,44]$. Agglomeration can also alleviate toxicity issues. It may mask defective surfaces from the cellular environment and, consequently, lessen the formation of reactive oxygen species (ROS) or preclude leaching of toxic components, such as $\mathrm{Cd}^{2+}$. Human liver and kidney cells (HepG2 and Hek293) were selected here because QD sequestration (in liver) and elimination by kidney were suggested previously as the critical sites of potential cytotoxic effects by metallic nanostructures, particularly those containing cadmium or mercury [36]. Given that the internalization process of nanomaterials varies depending on the properties of the cell plasma membrane, it is important to investigate several 
cell types under comparable conditions, since the constitution of the cell membrane depends on the cell type and, for a given cell line, on the physiological and pathological conditions.

\section{Conclusions}

This study demonstrates that the agglomeration state of nanoparticles, in this case quantum dots, in biological media can be determined reliably and rapidly by AF4 equipped with suitable detectors. This technique provides in a single experiment the size and size distribution of QDs agglomerates in serum-containing cell culture media. It also permits the quantitative determination of the extent of agglomeration and the relative concentration of isolated nanoparticles and agglomerates. These data cannot be obtained readily and/or reliably by other techniques, such as size exclusion chromatography, or by batch-mode DLS or SLS, which suffer from the unavoidable interference of serum proteins. Such interference is eliminated in the case of AF4 since the separation of the mixtures is performed in the absence of solid support, and particles are fractionated by size prior to characterization. Findings of QD internalization in different cell lines and data from AF4 strongly support the use of AF4 as a tool which defines, by a single measurement, the biological identity of nanoparticles as they encounter cells, an important piece of data for mechanistic in vitro studies related to the toxicity of nanoparticles. Given the broad size, shape, and composition of nanoparticles, which can be fractionated by AF4, our results suggest that the technique is applicable to most potentially harmful nanomaterials. Moreover, the combination of mass spectrometry proteomic analyses with AF4 separation may be a powerful approach to enhance the understanding of nanoparticle agglomeration in the presence of intra-cellular and extracellular proteins, and possible nanoparticle-induced changes of cellular functions related to their toxicity.

\section{Conflict of interest}

The authors report no conflict of interest. The authors alone are responsible for the content and writing of the paper.

\section{Acknowledgments}

The authors would like to thank Dr. Madjid Hadioui (Prof. Wilkinson's group, Université de Montréal, Canada) for performing the ICP-MS measurements. The work was supported in part by a grant of the World Premier International Research Center Initiative (WPI) MEXT, Japan to FMW and by a grant of the Canadian Institutes of Health Research (CIHR, MOP-119425) awarded to DM.

\section{Appendix A. Supplementary material}

Supplementary data associated with this article can be found, in the online version, at http://dx.doi.org/10.1016/j.ejpb.2014.12.019.

\section{References}

[1] N.A. Al-Hajaj, A. Moquin, K.D. Neibert, G.M. Soliman, F.M. Winnik, D. Maysinger, Short ligands affect modes of QD uptake and elimination in human cells, ACS Nano 5 (2011) 4909-4918.

[2] A. Albanese, W.C.W. Chan, Effect of gold nanoparticle aggregation on cell uptake and toxicity, ACS Nano 5 (2011) 5478-5489.

[3] Z.E. Allouni, M.R. Cimpan, P.J. Hol, T. Skodvin, N.R. Gjerdet, Agglomeration and sedimentation of $\mathrm{TiO}_{2}$ nanoparticles in cell culture medium, Colloids Surf. B Biointerf. 68 (2009) 83-87.

[4] L. Calzolai, D. Gilliland, C.P. Garcia, F. Rossi, Separation and characterization of gold nanoparticle mixtures by flow-field-flow fractionation, J. Chromatogr. A 1218 (2011) 4234-4239.

[5] T.J. Cho, V.A. Hackley, Fractionation and characterization of gold nanoparticles in aqueous solution: asymmetric-flow field flow fractionation with MALS, DLS, and UV-Vis detection, Anal. Bioanal. Chem. 398 (2010) 2003-2018.

[6] A.R. Clapp, E.R. Goldman, H. Mattoussi, Capping of CdSe-ZnS quantum dots with DHLA and subsequent conjugation with proteins, Nat. Protoc. 1 (2006) 1258-1266.

[7] J. Cohen, G. Deloid, G. Pyrgiotakis, P. Demokritou, Interactions of engineered nanomaterials in physiological media and implications for in vitro dosimetry, Nanotoxicology 7 (2013) 417-431.

[8] B. Dubertret, P. Skourides, D.J. Norris, V. Noireaux, A.H. Brivanlou, A. Libchaber, In vivo imaging of quantum dots encapsulated in phospholipid micelles, Science 298 (2002) 1759-1762.

[9] R. Duncan, S.C. Richardson, Endocytosis and intracellular trafficking as gateways for nanomedicine delivery: opportunities and challenges, Mol. Pharm. 9 (2012) 2380-2402.

[10] D.L. Elbert, J.A. Hubbell, Surface treatments of polymers for biocompatibility, Annu. Rev. Mater. Sci. 26 (1996). pp. 365-394.

[11] B.N. Feltis, S.J. O’keefe, A.J. Harford, T.J. Piva, T.W. Turney, P.F. Wright, Independent cytotoxic and inflammatory responses to zinc oxide nanoparticles in human monocytes and macrophages, Nanotoxicology 6 (2012) 757-765.

[12] W. Fraunhofer, G. Winter, The use of asymmetrical flow field-flow fractionation in pharmaceutics and biopharmaceutics, Eur. J. Pharm. Biopharm. 58 (2004) 369-383.

[13] X. Gao, L. Yang, J.A. Petros, F.F. Marshall, J.W. Simons, S. Nie, In vivo molecular and cellular imaging with quantum dots, Curr. Opin. Biotechnol. 16 (2005) 6372.

[14] M. Gaumet, A. Vargas, R. Gurny, F. Delie, Nanoparticles for drug delivery: the need for precision in reporting particle size parameters, Eur. J. Pharm. Biopharm. 69 (2008) 1-9.

[15] M. Geiser, M. Casaulta, B. Kupferschmid, H. Schulz, M. Semmler-Behnke, W. Kreyling, The role of macrophages in the clearance of inhaled ultrafine titanium dioxide particles, Am. J. Respir. Cell Mol. Biol. 38 (2008) 371-376.

[16] H. Hagendorfer, R. Kaegi, M. Parlinska, B. Sinnet, C. Ludwig, A. Ulrich, Characterization of silver nanoparticle products using asymmetric flow field flow fractionation with a multidetector approach - a comparison to transmission electron microscopy and batch dynamic light scattering, Anal. Chem. 84 (2012) 2678-2685.

[17] M. Hassellov, J.W. Readman, J.F. Ranville, K. Tiede, Nanoparticle analysis and characterization methodologies in environmental risk assessment of engineered nanoparticles, Ecotoxicology 17 (2008) 344-361.

[18] W.A. Hild, M. Breunig, A. Goepferich, Quantum dots - nano-sized probes for the exploration of cellular and intracellular targeting, Eur. J. Pharm. Biopharm. 68 (2008) 153-168.

[19] V. Hirsch, C. Kinnear, L. Rodriguez-Lorenzo, C.A. Monnier, B. RothenRutishauser, S. Balog, A. Petri-Fink, In vitro dosimetry of agglomerates, Nanoscale 6 (2014) 7325-7331.

[20] N. Hondow, R. Brydson, P. Wang, M. Holton, M.R. Brown, P. Rees, H. Summers, A. Brown, Quantitative characterization of nanoparticle agglomeration within biological media, J. Nanopart. Res. 14 (2012) 1-15.

[21] T.-G. Iversen, T. Skotland, K. Sandvig, Endocytosis and intracellular transport of nanoparticles: present knowledge and need for future studies, Nano Today 6 (2011) 176-185.

[22] M. Kendall, P. Ding, K. Kendall, Particle and nanoparticle interactions with fibrinogen: the importance of aggregation in nanotoxicology, Nanotoxicology 5 (2011) 55-65.

[23] S. Linse, C. Cabaleiro-Lago, W.F. Xue, I. Lynch, S. Lindman, E. Thulin, S.E. Radford, K.A. Dawson, Nucleation of protein fibrillation by nanoparticles, Proc. Natl. Acad. Sci. USA 104 (2007) 8691-8696.

[24] I. Lynch, K.A. Dawson, Protein-nanoparticle interactions, Nano Today 3 (2008) 40-47.

[25] H. Mattoussi, J.M. Mauro, E.R. Goldman, G.P. Anderson, V.C. Sundar, F.V. Mikulec, M.G. Bawendi, Self-assembly of CdSe-ZnS quantum dot bioconjugates using an engineered recombinant protein, J. Am. Chem. Soc. 122 (2000) 12142-12150.

[26] B.C. Mei, K. Susumu, I.L. Medintz, H. Mattoussi, Polyethylene glycol-based bidentate ligands to enhance quantum dot and gold nanoparticle stability in biological media, Nat. Protoc. 4 (2009) 412-423.

[27] T. Meißner, A. Potthoff, V. Richter, 2009. Suspension characterization as important key for toxicological investigations. JPCS 170:2012.

[28] X. Michalet, F.F. Pinaud, L.A. Bentolila, J.M. Tsay, S. Doose, J.J. Li, G. Sundaresan, A.M. Wu, S.S. Gambhir, S. Weiss, Quantum dots for live cells, in vivo imaging, and diagnostics, Science 307 (2005) 538-544.

[29] R.C. Murdock, L. Braydich-Stolle, A.M. Schrand, J.J. Schlager, S.M. Hussain, Characterization of nanomaterial dispersion in solution prior to in vitro exposure using dynamic light scattering technique, Toxicol. Sci. 101 (2008) 239-253.

[30] I. Nabiev, S. Mitchell, A. Davies, Y. Williams, D. Kelleher, R. Moore, Y.K. Gun'ko, S. Byrne, Y.P. Rakovich, J.F. Donegan, et al., Nonfunctionalized nanocrystals can exploit a cell's active transport machinery delivering them to specific nuclear and cytoplasmic compartments, Nano Lett. 7 (2007) 3452-3461.

[31] A. Noel, K. Maghni, Y. Cloutier, C. Dion, K.J. Wilkinson, S. Halle, R. Tardif, G. Truchon, Effects of inhaled nano- $\mathrm{TiO}_{2}$ aerosols showing two distinct agglomeration states on rat lungs, Toxicol. Lett. 214 (2012) 109-119.

[32] N. Ould-Moussa, M. Safi, M.A. Guedeau-Boudeville, D. Montero, H. Conjeaud, J.F. Berret, In vitro toxicity of nanoceria: effect of coating and stability in biofluids, Nanotoxicology 8 (2014) 799-811. 
[33] J.M. Pettibone, J. Gigault, V.A. Hackley, Discriminating the states of matter in metallic nanoparticle transformations: what are we missing?, ACS Nano 7 (2013) 2491-2499

[34] T. Pons, N. Lequeux, B. Mahler, S. Sasnouski, A. Fragola, B. Dubertret, Synthesis of near-infrared-emitting, water-soluble CdTeSe/CdZnS core/shell quantum dots, Chem. Mater. 21 (2009) 1418-1424

[35] T. Rameshwar, S. Samal, S. Lee, S. Kim, J. Cho, I.S. Kim, Determination of the size of water-soluble nanoparticles and quantum dots by field-flow fractionation, J. Nanosci. Nanotechnol. 6 (2006) 2461-2467.

[36] B.A. Rzigalinski, J.S. Strobl, Cadmium-containing nanoparticles: perspectives on pharmacology and toxicology of quantum dots, Toxicol. Appl. Pharmacol. 238 (2009) 280-288.

[37] B. Schmidt, K. Loeschner, N. Hadrup, A. Mortensen, J.J. Sloth, C.B. Koch, E.H. Larsen, Quantitative characterization of gold nanoparticles by field-flow fractionation coupled online with light scattering detection and inductively coupled plasma mass spectrometry, Anal. Chem. 83 (2011) 2461-2468.

[38] C. Schulze, A. Kroll, C.-M. Lehr, U.F. Schäfer, K. Becker, J. Schnekenburger, C. Schulze Isfort, R. Landsiedel, W. Wohlleben, Not ready to use - overcoming pitfalls when dispersing nanoparticles in physiological media, Nanotoxicology 2 (2008) 51-61.

[39] J.S. Taurozzi, V.A. Hackley, M.R. Wiesner, Ultrasonic dispersion of nanoparticles for environmental, health and safety assessment-issues and recommendations, Nanotoxicology 5 (2011) 711-729.

[40] K.G. Wahlund, J.C. Giddings, Properties of an asymmetrical flow field-flow fractionation channel having one permeable wall, Anal. Chem. 59 (1987) 1332-1339.

[41] D. Walczyk, F.B. Bombelli, M.P. Monopoli, I. Lynch, K.A. Dawson, What the cell "sees" in bionanoscience, J. Am. Chem. Soc. 132 (2010) 5761-5768.
[42] C.D. Walkey, J.B. Olsen, H. Guo, A. Emili, W.C.W. Chan, Nanoparticle size and surface chemistry determine serum protein adsorption and macrophage uptake, J. Am. Chem. Soc. 134 (2012) 2139-2147.

[43] C.D. Walkey, J.B. Olsen, F. Song, R. Liu, H. Guo, W. Olsen, Y. Cohen, A. Emili, W.C. Chan, Protein corona fingerprinting predicts the cell association of gold nanoparticles, ACS Nano 8 (2014) 2439-2455.

[44] M.A. Wells, A. Abid, I.M. Kennedy, A.I. Barakat, Serum proteins prevent aggregation of $\mathrm{Fe}_{2} \mathrm{O}_{3}$ and $\mathrm{ZnO}$ nanoparticles, Nanotoxicology 6 (2012) 837 846.

[45] W.H. Wu, X. Sun, Y.P. Yu, J. Hu, L. Zhao, Q. Liu, Y.F. Zhao, Y.M. Li, TiO nanoparticles promote beta-amyloid fibrillation in vitro, Biochem. Biophys. Res. Commun. 373 (2008) 315-318.

[46] Y. Xiao, S.P. Forry, X. Gao, R.D. Holbrook, W.G. Telford, A. Tona, Dynamics and mechanisms of quantum dot nanoparticle cellular uptake, J. Nanobiotechnol. 8 (2010) 13.

[47] L. Yang, L. Shang, G.U. Nienhaus, Mechanistic aspects of fluorescent gold nanocluster internalization by live HeLa cells, Nanoscale 5 (2013) 1537-1543.

[48] W.W. Yu, L.H. Qu, W.Z. Guo, X.G. Peng, Experimental determination of the extinction coefficient of CdTe, CdSe, and CdS nanocrystals, Chem. Mater. 15 (2003) 2854-2860.

[49] A. Zattoni, D.C. Rambaldi, P. Reschiglian, M. Melucci, S. Krol, A.M. Garcia, A. Sanz-Medel, D. Roessner, C. Johann, Asymmetrical flow field-flow fractionation with multi-angle light scattering detection for the analysis of structured nanoparticles, J. Chromatogr. A 1216 (2009) 9106-9112.

[50] L.W. Zhang, N.A. Monteiro-Riviere, Mechanisms of quantum dot nanoparticle cellular uptake, Toxicol. Sci. 110 (2009) 138-155.

[51] Y. Zhang, A. Clapp, Overview of stabilizing ligands for biocompatible quantum dot nanocrystals, Sensors 11 (2011) 11036-11055. 